\title{
Expression Changes of Some miRNAs Patterns (miR-124a, miR-137, miR-373) in Patients with Invasive Breast Cancer Before and After Neoadjuvant Chemotherapy
}

Ryspayeva Dinara ( $\square$ ryspayeva1@gmail.com )

National Cancer Institute

Halytskiy Volodymyr

Palladin Institute of Biochemistry of the National Academy of Sciences of Ukraine

Inomistova Mariia

National Cancer Institute

Drevytska Tetyana

Gyrianov Vitalyi

O.O.Bogomolets National Medical University

\section{Research Article}

Keywords: resectable breast cancer, neoadjuvant chemotherapy, tumor tissue, adjacent non-tumor tissue, expression miRNAs, miR-124a, miR-137, miR-34a, miR-155, miR-373

Posted Date: July 14th, 2021

DOI: https://doi.org/10.21203/rs.3.rs-647963/v1

License: (c) (i) This work is licensed under a Creative Commons Attribution 4.0 International License. Read Full License 


\section{Abstract}

\section{Background:}

Dysregulated miRNA expression has been found in nearly all the stages of cancer process compared to normal tissue. Certain miRNAs expression level is closely related to the histopathological parameters and molecular subtypes of breast cancer (BC) as well as the response to treatment and prognosis.

\section{Methods:}

This prospective research has evaluated the level of expression of 5 miRNAs (miR-124a, miR-137, miR34a, miR-155, miR-373) in the primary tumor before and after chemotherapy in patients with operable BC. The patients received 2-4 cycles neoadjuvant chemotherapy (NACT) and then underwent surgery mastectomy or breast conservation with axillary lymph node dissection. Assessment of all mRNAs was analyzed in selected pathology specimens obtained during the biopsy and the targeted area taken by the surgery.

\section{Results:}

A total of 34 patients were treated between February 2016 and December 2017 with median age of 53 years $(23-75) .70 .6 \%$ were HR-positive and $29.4 \%$ were HR-negative.

AntioncomiR-124a expression was mostly downregulated in primary BC tissue $(p=0.001)$. The restored expression of miR-124 ( $p=0.004)$ was observed after chemotherapy having reached the indistinguishable level from non-tumor tissue.

The dynamics of miR-373 expression considered to be oncomiR was a 7.7-fold decrease level in the primary tumor with continued suppress of expression compared to non-tumor tissue $(p=0.006)$.

The expression levels of miR-34a, miR-137, miR-155 in the pathology specimens did not show the differences compared to the surrounding non-tumor tissue ( $p>0.05)$.

The difference in the dynamics of the two suppressor miRNAs (miR-124a and miR-137) expression in HRpositive BC was identified. While the expression of miR-124a in the tumor tissue increases after NACT $(p=0.021)$, the expression of miR-137 continues to decrease $(p=0.041)$ and does not differ from the levels of non-tumor tissue $(p=0.146)$.

There was no relation between the expression of the 5 studied miRNAs with histopathological differentiation of the primary tumor and the pathomorphological response after NACT. A Cox proportional hazards model did not confirm the relationship between miRNAs expression level neither in primary tumor nor in clinicopathological BC characteristics.

\section{Conclusions:}


The differential regulatory effects of miRNA cannot be explained by the differences in expression alone as paradoxical behavior of miR-373 shows. Taking into account the heterogeneity of the breast tumor, the detected inconsistency of changes in the expression of two suppressor miRNAs (miR-124a and miR-137) can be explained by their different regulatory functions. An accurate picture of the functioning of microRNA requires further research due to its dynamic nature.

\section{Introduction}

Breast cancer $(\mathrm{BC})$ is a heterogeneous disease with different inter- and intratumoral heterogeneity. $\mathrm{BC}$ is commonly classified into the following intrinsic subtypes: luminal $A$, luminal $B$, human epidermal growth factor receptor 2 (HER2/neu)-enriched and basal-like presented as triple-negative (TN). HR-positive (hormone receptor) BC includes luminal A, luminal $B$ and luminal B HER2-positive. HR-negative BC is represented by HER2-enriched and TN subtypes [1].

In recent years, microRNAs attract significant interest due to their regulatory involvement in cancer initiation, progression and metastasis [2-4]. Studies have shown that certain miRNA signatures exhibit differential expression and correlate with tumor aggressiveness, response to the therapy and outcome in different tumors [5-7].

In addition, certain miRNAs expression level is closely related to the histopathological parameters and molecular subtypes of $B C$ as well as the response to treatment and prognosis [8-13]. It has been shown that some microRNAs in $\mathrm{BC}$ activate the functions of oncogenes while others stimulate tumor suppressors [4]. Despite of increasing the number of identified miRNAs in humans, their role in the regulation of gene expression is partially established on account of a large number of targets. Therefore, the exact relationship between some miRNAs and the biological behavior of $\mathrm{BC}$ remains unclear and requires further research.

miR-34 performs as a tumor suppressor preventing the development of various types of cancer [6, 14]. A decrease in miR-34 levels activates epithelial to mesenchymal transition (EMT) programs, in which the Snail1 pathway serves as a dominant effector of the invasive BC cell phenotype [15]. Downregulation of miR-34a is associated with an unfavourable clinical outcome and prognosis of the disease [16]. High concentrations of miR-34a in TNBC, the responsibility of overexpression of miR-34a for poor prognostic factors (positive nodal status, high tumor grade, HER2-positivity, high proliferation rate) and the occurrence of drug resistance to docetaxel in BC cells are described by other authors [17-19].

Other oncosuppressive miR-124 is characterized by high expression in normal tissues [20] and low expression in most tumors [21-26]. Suppression of miRNA-124 expression in the tumor appeared to be an independent predictor of poor survival $[21,22,25,27,28]$.

Although different miR-124 targets are known in various tissues and tumor types [22-25, 29], the regulation of miR-124 expression is only partially deciphered nowadays. Recent studies have shown that 
miR-124 downregulation is associated with tumor progression and may be a diagnostic and prognostic biomarker $[20,21,26]$.

miR-137 downregulation has been associated frequently with various types of cancer and to a great extent with poor prognostic clinico-pathological parameters and decreased overall survival (OS) [30-35]. Studies have shown that miR-137 often performs as a tumor suppressor in gastric [36-39], colorectal cancer [40, 41], cholangiocarcinoma [42], kidney [32, 33], ovarian [43], endometrial [44] and non-small cell lung cancer $[45,46]$, melanoma $[47,48]$, triple-negative breast cancer (TNBC) $[49,50]$. Recent studies, however, have shown that miR-137 is upregulated in lung [51] and bladder cancer [52].

The relation between miR-137 and BC has been established in the last few years but the data is conflicting. The levels of this miR were remarkably low in unfavourable TNBC [49,50], and at the same time there is an evidence of high levels of miR-137 in BC samples associated with a decrease patient survival [53].

miR-155 is upregulated in tumor tissue and performs as an oncogenic miRNA (oncomiR) in kidney [54], gastric cancer [55] and BC $[14,56])$.

The meta-analysis has shown that the determination of miR-155 expression level has a diagnostic potential for detection of $\mathrm{BC}$ [57]. miR-155 upregulation was reported as an indicator of breast tumor invasiveness, late-stage BC with lymph node metastases, high grade and poor prognosis [56, 58, 59]. However, in another study miR-155 overexpression was determined protective and correlated with a better $\mathrm{BC}$ prognosis [60].

miR-373 was initially identified as a potential oncomiR involved in the development of human testicular germ cell tumors. It was noted previously that miR-373 expression was more common for embryonic tumors and was rare in somatic tumors [61].

Overexpression of miR-373 is associated with lymph node metastases in BC [62] and poor OS in squamous cell head and neck cancer [63]. At the same time the other studies have shown the suppressive role of miR-373 with contribution to tumor invasion [5, 64-66]. The patients with low miR-373 expression were found to have shorter OS and progression-free survival (PFS) [64].

The purpose of the present study is to determine the level of expression of 5 miRNAs (miR-124a, miR-137, miR-34a, miR-155, miR-373) in the primary tumor before and after chemotherapy in patients with resectable $\mathrm{BC}$ and assess the clinical significance of the results.

\section{Methods}

A total of 34 patients ( 318 years old) with a confirmed histologic diagnosis of breast cancer were enrolled in the study treated at National Cancer Institute (Ukraine) between May 2016 and December 2017. All the patients gave written informed consent obtained before the procedure or treatment. The present study was approved by the local Ethics Committee. 
The average age of the patients was 53 years old (the range from 23 to 75$)$. The half of patients (50\%) were diagnosed with locally advanced (T3 N1-3 M0) disease (Table 1). The majority of patients were HRpositive $(n=24 ; 70.6 \%)$ and a smaller part - HR-negative $(n=10 ; 29.4 \%)$.

The clinical stage of TNM was assessed by physical examination and mammography, ultrasound of the breast, axilla and abdomen, chest X-ray or computed tomography scan. Demographic data and medical history were collected from medical records.

Prior to treatment, ultrasound-guided core needle biopsies using an automatic biopsy instrument (Fast Gun, Sterylab) were collected from 34 female patients. Three - four specimens were obtained from each lesion, half of them were enclosed in Eppendorf tubes containing DNA/RNA Shield reagent for sample preservation, then frozen at $-70^{\circ} \mathrm{C}$ for subsequent miRNAs profiling.

Patients received NACT intravenously a day in 21-day cycles. Preoperative treatment was 2-4 cycles. The following NACT regimens were used: FAC (doxorubicin $50 \mathrm{mg} / \mathrm{m}^{2}$, cyclophosphamide $500 \mathrm{mg} / \mathrm{m}^{2}$, 5fluorouracil $500 \mathrm{mg} / \mathrm{m}^{2}$ ), AT (doxorubicin $50 \mathrm{mg} / \mathrm{m}^{2}$, docetaxel $75 \mathrm{mg} / \mathrm{m}^{2}$ or paclitaxel $175 \mathrm{mg} / \mathrm{m}^{2}$ ), AC (doxorubicin $60 \mathrm{mg} / \mathrm{m}^{2}$, cyclophosphamide $600 \mathrm{mg} / \mathrm{m}^{2}$ ) and TC regimen (docetaxel $75 \mathrm{mg} / \mathrm{m}^{2}$ plus cyclophosphamide $600 \mathrm{mg} / \mathrm{m}^{2}$ ).

All the patients following NACT underwent surgery - mastectomy or breast conservation with axilliary lymph node dissection. Specimens from tumor tissue of 34 patients and from 15 of them obtained paired adjacent non-tumor tissues were collected in Eppendorf tubes containing DNA/RNA Shield reagent and frozen at $-70^{\circ} \mathrm{C}$ for subsequent miRNAs profiling.

Paraffin-embedded tumor samples were evaluated by immunohistochemical staining analysis for estrogen receptor (ER) (clone EP1; Dako, Denmark), progesterone receptor (PgR) (clone PgR 636; Dako) and Ki-67 (clone MIB-1; Dako). HER2 status was evaluated by immunohistochemical or by fluorescence in situ hybridization according to the manufacturer's instructions.

All tumors were graded by the study pathologists according to Nottingham grading system. Pathological response in the postsurgery specimens was assessed by local pathologists using Miller-Payne scoring system with the evaluation of residual tumor cells. Pathological complete response was defined as noninvasive residual disease in breast tissue and auxillary lymph nodes after NACT as reported previously [67].

\section{Analysis of miRNA expression}

RT-qPCR (reverse transcription quantitative polymerase chain reaction) assays were performed to determine the expression level of miR-124a, miR-137, miR-34a, miR-155 and miR-373. Assessment of all mRNA was analyzed in a sample of tumor tissue obtained during the biopsy and the targeted area taken by the surgery. 
To isolate total RNA and microRNA, the NucleoSpin miRNA kit (Macherey-Nagel, Germany) was used according to the manufacturer's protocol.

The results were detected in real time using the 7500 Real-Time PCR System amplifier and a mixture of Universal PCR Master Mix reagents (Applied Biosystems, USA). TaqMan ${ }^{\circledR}$ MIRNA Assays primers (mmumiR-124a, hsa-miR-155, hsa-miR-34a, hsa-miR-373, mmu-miR-137) were applied to detect microRNAs in the $\mathrm{q}-\mathrm{PCR}$ reaction.

The following temperature regimen was taken for the PCR reaction: activation of AmpliTaq Gold DNA polymerase at $50^{\circ} \mathrm{C}$ for 2 minutes, primary denaturation $95^{\circ} \mathrm{C}$ for 10 minutes, accumulation of the amplification product for 40 cycles $95^{\circ} \mathrm{C}-15$ seconds, $60^{\circ} \mathrm{C}-60$ seconds.

The Ct method was made to normalize the expression levels of target genes by correcting the difference in the amount of cDNA relatively to the endogenous control for microRNA U6B (TaqMan ${ }^{\circledR}$ MicroRNA Control RNU6B). miRNA expression level was calculated by the $\triangle \mathrm{Ct}$ method.

\section{Statistical analysis}

Statistical data were analyzed in the same patients before and after NACT. In order to control the results, paired adjacent non-tumor tissues in the postoperative specimens were collected from the same patients.

Pearson test $\chi 2$ (for qualitative variables) and Wilcoxon test (for quantitative variables) were used to compare clinical and pathological data in the groups before and after treatment. A Mann-Whitney test was applied to compare the differences of HR-positive and HR-negative BC. A Kruskal-Wallis test and post hoc analysis (Dunn's test) were performed to compare the differences in miRNA levels of more than two groups.

Multivariate analysis of the prognosis factors with a Cox proportional hazards model was made. $P<0.05$ was considered to indicate a statistically significant difference.

\section{Results}

The clinicopathological characteristics before and after treatment listed in Table 1 summarize the stage of disease and index of proliferation. As shown in Table 1, the increase in the number of lymph nodesnegative patients from $11.8 \%$ to $29.4 \%$ after NACT was statistically insignificant $(p=0.289)$. There was also no statistically significant difference in the dynamics of Ki 67 values and in the tumor tissue before and after NACT $(p=0.855)$.

Among all the miRNAs studied antioncomiR-124a expression was the most significantly $(p=0.001)$ downregulated in primary BC tissues (Table 2). After NACT a significant increase in miR-124a expression $(p=0.004)$ is observed in the tumor reaching a level that is indistinguishable from non-tumor tissue. 
The dynamics of miR-373 expression considered as oncomiR seems to be interesting. After the treatment, there was a 7.7-fold decrease in miR-373 expression levels in the primary tumor with continued suppress of expression compared to non-tumor tissue $(\mathrm{p}=0.006)$.

The expression level of the remaining miRNAs (miR-34a, miR-137, miR-155) in the tumor tissue did not show the differences before and after treatment compared to the surrounding non-tumor tissue $(p>0.05)$.

Subgroup analysis revealed a difference of two suppressor miRNAs in HR-positive BC patients. The dynamics of the expression levels of suppressors miR-124a and miR-137 in HR-positive BC is shown in the figures (Fig. 1, 2). While the expression of miR-124a in the tumor tissue significantly increases after the treatment $(p=0.021)$ the expression of miR-137 continues significantly to decrease $(p=0.041)$ and does not differ from the levels of non-tumor tissue $(p=0.146)$. At the same time the differences in the dynamics of expression of the studied miRNAs in the tumor tissue of patients with HR-negative BC were not found.

Besides there was no correlation between the expression of the 5 studied miRNAs with histopathological grading of the primary tumor (Table 3 ) and pathomorphological response after NACT.

Composite measure of recurrence risk for each patient was determined from a Cox model incorporating age, stage, tumor size, nodal status, Ki-67 index and expression levels of 5 investigated miRNAs (Table 4). Regarding to multivariate analysis of the prognostic factors with a Cox proportional model the relationship between miRNAs expression level in primary tumor and clinicopathological BC characteristics was not confirmed.

\section{Discussion}

In the era of personalized treatment miRNAs stand out among predictive markers as to their potential clinical application. In almost all the stages of the cancer process (cell cycle, apoptosis, invasion, angiogenesis), dysregulated miRNA expression has been found when compared to normal tissue $[3,4,12$, $14,68]$.

The set and expression level of miRNAs and the corresponding DNA methylation pattern are strictly controlled and highly specific for each normal tissue including the epithelium and stroma. In cancer these patterns undergo profound changes that occur both in the primary tumor tissue and in the adjacent microenvironment $[3,12,14]$.

The levels of expression of several miRNAs were determined in this study and was made an attempt to identify the relationship with the clinicopathologic properties of $B C$ and the response to NACT.

\section{miR-34a}

The potential links between oncogenic processes, miR-34a expression and the regulation of the activity of the Snail1 transcription factor responsible for EMT, cell migration, invasive growth and metastasis have 
been established previously [15].

The changes of miR-34a expression either in the primary tumor tissue or after the treatment in patients with $B C$ were not detected in the research. An explanation to that is the analysis carried out was of earlystage, non-metastatic $B C$ and accordingly prior to the reactivation of the EMT-like programs.

\section{miR-124}

The ability of oncosupressor miR-124 to regulate the proliferation of tumor cells is significant for a therapeutic strategy [29]. The overexpression of miR-124 can inhibit the proliferation of tumor cells and reduce tumor growth increasing the sensitivity of cells to chemotherapy in vitro $[21,23,29]$.

The restored expression of miR-124 was observed after chemotherapy $(p=0.004)$ in the general study group and in the HR-positive BC subgroup $(p=0.021)$.

Earlier strong evidence was presented that high miR-124 expression may be an independent marker of a favourable prognosis in many oncological diseases including invasive $B C[20,21,69]$, while reduced miR124 expression correlated with lymph node metastases and low OS [27, 28].

\section{miR-137}

miR-137 negatively regulates a wide range of downstream targets in various types of cancer and can target multiple transcripts like miR-124 [41, 70].

A decrease in the level of tumor supressor miR-137 expression after NACT in HR-positive BC patients $(p=0.041)$ have been found in current research. It is known that a number of microRNAs are expressed differently in the luminal and basal subtypes of $\mathrm{BC}$ reflecting their epithelial and myoepithelial nature respectively $[2,10,71]$. Other researchers noted that the expression levels of most miRNAs were mostly lower in HR-negative BC [50, 71].

The continued decrease in miR-137 expression after NACT is more characteristic for behaviour of oncomiR. The frequency of aberrant methylation leading to downregulation miR-137 and affecting oncogenesis is only $37 \%$ and, therefore, it is not always possible to observe behavior of miR-137 as a oncosuppressor [72].

On the other hand, the expression of the estrogen-related receptor a (ERRa), which plays an important role in the progression of $\mathrm{BC}$, is regulated by $\mathrm{miR}-137$. There is an inverse relationship between the level of miR-137 and the expression of ERRa, that is, with a decrease level in miR-137 there will be a dysregulation of ERRa which may contribute to BC tumorigenesis [70].

In this case $B C$ cells respond to systemic treatment by decreasing miR-137 expression. It can be assumed that miR-137 may also function as an oncomiRNA depending on the context of the transformed cells after NACT. 
A reasonable explanation for this phenomenon may be the complexity of the regulation of gene transcription. The transcription of a particular gene is controlled by several transcription factors and the change in miRNA expression level is the result of the dynamic balance of each component in this complex [73]. As mentioned above a single specific microRNA can also target more than one transcript. That means ERRa is not the only transcription factor targeted by miR-137 but, moreover, miR-137 may play different roles depending on the different tumor microenvironments.

Some microRNAs have large mismatches between expression levels and activity to repress their targets. As noted before there is a very weak positive correlation between miRNA expression and their regulatory effects [71]. It is likely that miR-137 is one of them and the ability of miRNAs to repress their targets may not be reflected in their expression level.

\section{miR-155}

The oncogenic role of this microRNA (miR-155) in primary BC has been previously shown in a number of studies $[58,59,74]$ and significant suppression of this microRNA was observed in patients with metastatic disease [19]. The level of miR-155 overexpression decreased after surgical resection, chemoor radiotherapy and correlated with a decrease in tumor volume $[56,75]$. Other studies have shown that overexpression of miR-155 predicts resistance to systemic BC therapy $[11,76]$.

No significant changes in the expression of miR-155 in tumor tissue during treatment have been found $(p=0.741)$.

\section{miR-373}

MiR-373 expression levels may reflect different degrees of activity and the ability of tumours to metastasize [62]. Thus, overexpression of miR-372/373 contributes to induction of the invasive phenotype by targeting CD44 [14, 62, 77]. According to the other data overexpression of miR-373 on the contrary can suppress cell proliferation and tumor growth [78]. So, miR-373 suppressed or elevated is involved to the oncogenesis of various types of tumors [79]. It is not difficult to understand the phenomenon of paradoxical behavior of miR-373 taking into account the heterogeneity of the tumor [79].

Can the study of clinical samples shed some light to the functions of miR-373? The data remains inconsistent. On the one side, increasing miR-373 expression was positively correlated with a more malignant phenotype, HER2/neu-positive BC, lymph node involvement and the late stages of the disease[62, 80, 81]. On the other side, miR-373 expression was negatively correlated with lymph node metastases and reduced levels of this microRNA in the serum of HER2/neu-positive BC patients $[19,82]$.

A suppression of miR-373 expression in the primary tumor and further level decrease after NACT were found in the present study $(p=0.006)$. The suppression of miR-373 on the background of CT may underlie some antitumor effects also increase radiosensitivity and inhibit the migration and invasion of tumor cells [83]. 
Other authors, on the contrary, demonstrate the effect of NACT on serum concentrations of miR-373 in the form of an increase in its levels [81]. According to the authors [5], genome-wide erasure of DNA methylation occurs due to the influence of cytotoxic therapy which leads to re-expression of miR-373 and, as a result, to an apoptotic effect in cancer cells..

Indeed, studies show that miR-373 can have both oncogenic activity and exhibit the properties of tumor suppressors [79].

\section{Conclusions}

In conclusion it should be said that the miRNA regulatory network is quite complex and the differential regulatory effects of miRNA cannot be explained by differences in expression alone. The result of the action of some microRNA clusters can be a multiple attack on a common target or a coordinated control of a number of independent targets [73]. So far, most of the role of miRNAs is understood only from studies on cell culture models and a limited number of clinical observations.

Taking into consideration the heterogeneity of the breast tumor the detected changes in miRNA expression levels between HR-positive and HR-negative may depend on the cellular context and preexisting genetic or epigenetic damage. The inconsistency of changes in the expression of two suppressor miRNAs (miR-124a and miR-137) may be due to their different regulatory functions. The phenomenon of paradoxical behaviour of miR-373 can be explained by the deregulating one particular miRNA which depends on the genetic characteristics of the tumor cells and the tumor microenvironment.

An accurate picture of microRNA functioning requires further research in order to explain its dynamic nature.

\section{Declarations}

\section{Ethics approval and consent to participate}

The study was conducted to the guidelines of the Declaration of Helsinki and approved by the Ethics Committee of National Cancer Institute (\#81 from 05 May 2016) and all patients gave their informed consent for inclusion before the procedure or treatment.

\section{Consent for publication}

Not applicable.

\section{Competing interests}

The authors declare that they have no competing interests

\section{Author details}


${ }^{1}$ National Cancer Institute, Kyiv 03022, Ukraine

${ }^{2}$ Palladin Institute of Biochemistry of the National Academy of Sciences of Ukraine, Kyiv, 01054, Ukraine

${ }^{3}$ O.O.Bogomolets Institute of Physiology NASU, 01024 Kyiv, Ukraine

${ }^{4}$ O.O.Bogomolets National Medical University, Kyiv 03115, Ukraine

Corresponding author: Ryspayeva Dinara, MD, PhD

Department of oncohematology and adjuvant treatment methods,

National Cancer Institute, Lomonosova str, 33/43, Kyiv, 03022, Ukraine

Phone: +38(050) 636-25-19, Fax: +38 (044) 259-02-73

e-mail: ryspayeva1@gmail.com

\section{Abbreviations}

BC - breast cancer; NACT - neoadjuvant chemotherapy; HER2/neu - human epidermal growth factor receptor 2; TN - triple-negative; TNBC - triple-negative breast cancer; HR - hormone receptor; EMT epithelial to mesenchymal transition; OS - overall survival; PFS - progression-free survival; ER estrogen receptor; PR - progesterone receptor; PCR - polymerase chain reaction; ERRa estrogen-related receptor $\mathrm{a}$.

\section{References}

1. Goldhirsch, A., et al., Personalizing the treatment of women with early breast cancer: highlights of the St Gallen International Expert Consensus on the Primary Therapy of Early Breast Cancer 2013. Annals of oncology : official journal of the European Society for Medical Oncology, 2013. 24(9): p. 2206-2223.

2. Kurozumi, S., et al., Recent trends in microRNA research into breast cancer with particular focus on the associations between microRNAs and intrinsic subtypes. Journal of human genetics, 2017. 62(1): $\mathrm{p}$. 15-24.

3. Lu, J., et al., MicroRNA expression profiles classify human cancers. Nature, 2005. 435(7043): p. 834-8.

4. Izumiya, M., et al., Systematic exploration of cancer-associated microRNA through functional screening assays. Cancer Science, 2011. 102(9): p. 1615-1621.

5. Adi Harel, S., et al., Reactivation of epigenetically silenced miR-512 and miR-373 sensitizes lung cancer cells to cisplatin and restricts tumor growth. Cell Death Differ, 2015. 22(8): p. 1328-40. 
6. Jiang, $\mathrm{H}$. , et al., MicroRNA-34a inhibits esophageal squamous cell carcinoma progression by targeting E2F5. J BUON, 2019. 24(6): p. 2514-2522.

7. Lima, C.R., C.C. Gomes, and M.F. Santos, Role of microRNAs in endocrine cancer metastasis. Molecular and Cellular Endocrinology, 2017. 456(C): p. 62-75.

8. Iorio, M.V., et al., MicroRNA gene expression deregulation in human breast cancer. Cancer Res, 2005. 65(16): p. 7065-70.

9. Ohzawa, $\mathrm{H}$. , et al., Usefulness of miRNA profiles for predicting pathological responses to neoadjuvant chemotherapy in patients with human epidermal growth factor receptor 2-positive breast cancer. Oncology letters, 2017. 13(3): p. 1731-1740.

10. Blenkiron, C., et al., MicroRNA expression profiling of human breast cancer identifies new markers of tumor subtype. Genome Biol, 2007. 8(10): p. R214.

11. Campos-Parra, A.D., et al., Micro-RNAs as Potential Predictors of Response to Breast Cancer Systemic Therapy: Future Clinical Implications. Int J Mol Sci, 2017. 18(6).

12. Takahashi, R.U., H. Miyazaki, and T. Ochiya, The Roles of MicroRNAs in Breast Cancer. Cancers (Basel), 2015. 7(2): p. 598-616.

13. Kolacinska, A., et al., Association of microRNAs and pathologic response to preoperative chemotherapy in triple negative breast cancer: preliminary report. Molecular biology reports, 2014. 41(5): p. 2851-2857.

14. Negrini, M., M.S. Nicoloso, and G.A. Calin, MicroRNAs and cancer-new paradigms in molecular oncology. Curr Opin Cell Biol, 2009. 21(3): p. 470-9.

15. Kim, N.H., et al., A p53/miRNA-34 axis regulates Snail1-dependent cancer cell epithelialmesenchymal transition. J Cell Biol, 2011. 195(3): p. 417-33.

16. Zhang, $\mathrm{H}$., et al., The prognostic value of miR-34a expression in completely resected gastric cancer: tumor recurrence and overall survival. International Journal of Clinical and Experimental Medicine, 2015. 8(2): p. 2635-2641.

17. Kastl, L., I. Brown, and A.C. Schofield, miRNA-34a is associated with docetaxel resistance in human breast cancer cells. Breast Cancer Res Treat, 2012. 131(2): p. 445-54.

18. Peurala, $\mathrm{H}$., et al., MiR-34a expression has an effect for lower risk of metastasis and associates with expression patterns predicting clinical outcome in breast cancer. PLoS One, 2011. 6(11): p. e26122.

19. Eichelser, C., et al., Deregulated Serum Concentrations of Circulating Cell-Free MicroRNAs miR-17, miR-34a, miR-155, and miR-373 in Human Breast Cancer Development and Progression. Clinical 
Chemistry, 2013. 59(10): p. 1489-1496.

20. Zhou, Z., et al. Role of MicroRNA-124 as a Prognostic Factor in Multiple Neoplasms: A MetaAnalysis. Disease markers, 2019. 2019, 1654780 DOI: 10.1155/2019/1654780.

21. Chen, Z., et al., miR-124 and miR-506 inhibit colorectal cancer progression by targeting DNMT3B and DNMT1. Oncotarget, 2015. 6(35): p. 38139-38150.

22. Huang, T.T., et al., SRGAP1, a crucial target of miR-340 and miR-124, functions as a potential oncogene in gastric tumorigenesis. Oncogene, 2018. 37(9): p. 1159-1174.

23. Yang, Q.H., et al., Inhibition of LHX2 by miR-124 suppresses cellular migration and invasion in nonsmall cell lung cancer. Oncology Letters, 2017. 14(3): p. 3429-3436.

24. Zhang, S., et al., CircRNA_0000502 promotes hepatocellular carcinoma metastasis and inhibits apoptosis through targeting microRNA-124. J BUON, 2019. 24(6): p. 2402-2410.

25. Zhao, Y., et al., MiR-124 acts as a tumor suppressor by inhibiting the expression of sphingosine kinase 1 and its downstream signaling in head and neck squamous cell carcinoma. Oncotarget, 2017. 8(15): p. 25005-25020.

26. Yuan, L., et al., MiR-124 inhibits invasion and induces apoptosis of ovarian cancer cells by targeting programmed cell death 6. Oncol Lett, 2017. 14(6): p. 7311-7317.

27. Dong, L.L., et al., Decreased expression of microRNA-124 is an independent unfavorable prognostic factor for patients with breast cancer. Diagn Pathol, 2015. 10: p. 45.

28. Zhang, L., et al., MicroRNA-124-3p directly targets PDCD6 to inhibit metastasis in breast cancer. Oncol Lett, 2018. 15(1): p. 984-990.

29. Han, Z.B., et al., MicroRNA-124 Suppresses Breast Cancer Cell Growth and Motility by Targeting CD151. Cellular Physiology and Biochemistry, 2013. 31(6): p. 823-832.

30. Zhang, $\mathrm{H}$., et al., MicroRNA-137 is negatively associated with clinical outcome and regulates tumor development through EZH2 in cervical cancer. J Cell Biochem, 2018. 119(1): p. 938-947.

31. Gu, Q., et al., Clinical Significance of MiR-137 Expression in Patients with Gastric Cancer After Radical Gastrectomy. Plos One, 2015. 10(11).

32. Zhang, $\mathrm{H}$. and H. Li, miR-137 inhibits renal cell carcinoma growth in vitro and in vivo. Oncology Letters, 2016. 12(1): p. 715-720.

33. Wang, M., et al., MiR-137 suppresses tumor growth and metastasis in clear cell renal cell carcinoma. Pharmacological reports : PR, 2018. 70(5): p. 963-971. 
34. Li, N., Low Expression of Mir-137 Predicts Poor Prognosis in Cutaneous Melanoma Patients. Medical Science Monitor, 2016. 22: p. 140-144.

35. Peres, J., et al., The tumour suppressor, miR-137, inhibits malignant melanoma migration by targetting the TBX3 transcription factor. Cancer Lett, 2017. 405: p. 111-119.

36. Chen, Q., et al., miR-137 Is Frequently Down-Regulated in Gastric Cancer and Is a Negative Regulator of Cdc42. Digestive Diseases and Sciences, 2011. 56(7): p. 2009-2016.

37. Cheng, Y., et al., miR-137 effects on gastric carcinogenesis are mediated by targeting Cox-2activated PI3K/AKT signaling pathway. Febs Letters, 2014. 588(17): p. 3274-3281.

38. Jin, W., et al., Epigenetic silencing of miR137 in gastric cancer. International Journal of Clinical and Experimental Medicine, 2016. 9(9): p. 17926-17932.

39. Steponaitiene, R., et al., Epigenetic Silencing of miR-137 is a Frequent Event in Gastric Carcinogenesis. Molecular Carcinogenesis, 2016. 55(4): p. 376-386.

40. Balaguer, F., et al., Epigenetic Silencing of miR-137 Is an Early Event in Colorectal Carcinogenesis. Cancer Research, 2010. 70(16): p. 6609-6618.

41. $\mathrm{Bi}, \mathrm{W}$.-P., M. Xia, and X.-J. Wang, miR-137 suppresses proliferation, migration and invasion of colon cancer cell lines by targeting TCF4. Oncology Letters, 2018. 15(6): p. 8744-8748.

42. Chen, T., et al., MicroRNA-137 suppresses the proliferation, migration and invasion of cholangiocarcinoma cells by targeting WNT2B. International Journal of Molecular Medicine, 2020. 45(3): p. 886-896.

43. Zhang, L., et al., MicroRNA-137 suppresses tumor growth in epithelial ovarian cancer in vitro and in vivo. Molecular Medicine Reports, 2015. 12(2): p. 3107-3114.

44. Zhang, W., et al., miR-137 is a tumor suppressor in endometrial cancer and is repressed by DNA hypermethylation. Laboratory Investigation, 2018. 98(11): p. 1397-1407.

45. Zhang, B., et al., microRNA-137 functions as a tumor suppressor in human non-small cell lung cancer by targeting SLC22A18. International Journal of Biological Macromolecules, 2015. 74: p. 111-118.

46. Min, L., et al., Aberrant microRNA-137 promoter methylation is associated with lymph node metastasis and poor clinical outcomes in non-small cell lung cancer. Oncology Letters, 2018. 15(5): p. 7744-7750.

47. Chang, X., et al., miR-137 suppresses tumor growth of malignant melanoma by targeting aurora kinase A. Biochemical and Biophysical Research Communications, 2016. 475(3): p. 251-256. 
48. Qi, J., et al., Mechanism of miR-137 regulating migration and invasion of melanoma cells by targeting PIK3R3 gene. Journal of Cellular Biochemistry, 2019. 120(5): p. 8393-8400.

49. Chae, Y.S., et al., Abstract P6-05-10: MicroRNA-137 inhibits cancer progression by targeting DEL-1 in triple negative breast cancer cells, MDA-MB-231. Cancer Research, 2019. 79(4 Supplement): p. P6-0510.

50. Chen, F., et al., MiR-137 Suppresses Triple-Negative Breast Cancer Stemness and Tumorigenesis by Perturbing BCL11A-DNMT1 Interaction. Cellular Physiology and Biochemistry, 2018. 47(5): p. 2147-2158.

51. Chang, T.H., et al., Upregulation of microRNA-137 expression by Slug promotes tumor invasion and metastasis of non-small cell lung cancer cells through suppression of TFAP2C. Cancer Lett, 2017. 402: p. 190-202.

52. Xiu, Y., et al., MicroRNA-137 Upregulation Increases Bladder Cancer Cell Proliferation and Invasion by Targeting PAQR3. Plos One, 2014. 9(10).

53. Ying, X., Y. Sun, and P. He, MicroRNA-137 inhibits BMP7 to enhance the epithelial-mesenchymal transition of breast cancer cells. Oncotarget, 2017. 8(11): p. 18348-18358.

54. Li, S., et al., microRNA-155 silencing inhibits proliferation and migration and induces apoptosis by upregulating BACH1 in renal cancer cells. Molecular Medicine Reports, 2012. 5(4): p. 949-954.

55. Shi, S.S., et al., Exosomal miR-155-5p promotes proliferation and migration of gastric cancer cells by inhibiting TP53INP1 expression. Pathology Research and Practice, 2020. 216(6): p. 8.

56. Farsinejad, S., et al., Expression of the circulating and the tissue microRNAs after surgery, chemotherapy, and radiotherapy in mice mammary tumor. Tumour Biol, 2016. 37(10): p. 14225-14234.

57. Hou, Y., et al., Appraising MicroRNA-155 as a Noninvasive Diagnostic Biomarker for Cancer Detection: A Meta-Analysis. Medicine (Baltimore), 2016. 95(2): p. e2450.

58. Hafez, M.M., et al., MicroRNAs and metastasis-related gene expression in Egyptian breast cancer patients. Asian Pac J Cancer Prev, 2012. 13(2): p. 591-8.

59. Kong, W., et al., Upregulation of miRNA-155 promotes tumour angiogenesis by targeting VHL and is associated with poor prognosis and triple-negative breast cancer. Oncogene, 2014. 33(6): p. 679-89.

60. Gasparini, P., et al., Protective role of miR-155 in breast cancer through RAD51 targeting impairs homologous recombination after irradiation. Proc Natl Acad Sci U S A, 2014. 111(12): p. 4536-41.

61. Voorhoeve, P.M., et al., A genetic screen implicates miRNA-372 and miRNA-373 as oncogenes in testicular germ cell tumors. Cell, 2006. 124(6): p. 1169-81. 
62. Huang, Q., et al., The microRNAs miR-373 and miR-520c promote tumour invasion and metastasis. Nat Cell Biol, 2008. 10(2): p. 202-10.

63. Tu, H.F., et al., Upregulation of miR-372 and -373 associates with lymph node metastasis and poor prognosis of oral carcinomas. Laryngoscope, 2015. 125(11): p. E365-70.

64. Jing, S.Y., et al., Down-expression of miR-373 predicts poor prognosis of glioma and could be a potential therapeutic target. Eur Rev Med Pharmacol Sci, 2017. 21(10): p. 2421-2425.

65. Nakata, K., et al., Micro RNA-373 is down-regulated in pancreatic cancer and inhibits cancer cell invasion. Ann Surg Oncol, 2014. 21 Suppl 4: p. S564-74.

66. Qu, Y.H., et al., [Effects of microRNA-373 on the proliferation and invasiveness of breast carcinoma and its mechanisms]. Zhonghua Yi Xue Za Zhi, 2017. 97(8): p. 603-607.

67. Ryspayeva, D., et al., Predictive factors of pathological response to neoadjuvant chemotherapy in patients with breast cancer. J BUON, 2020. 25(1): p. 168-175.

68. Landskroner-Eiger, S., I. Moneke, and W.C. Sessa, miRNAs as modulators of angiogenesis. Cold Spring Harb Perspect Med, 2013. 3(2): p. a006643.

69. Feng, T., et al., Evaluation of the Role of hsa-mir-124 in Predicting Clinical Outcome in Breast Invasive Carcinoma Based on Bioinformatics Analysis. Biomed Research International, 2020. 2020.

70. Zhao, Y., et al., MiR-137 Targets Estrogen-Related Receptor Alpha and Impairs the Proliferative and Migratory Capacity of Breast Cancer Cells. PLOS ONE, 2012. 7(6): p. e39102.

71. Cheng, C., et al., mRNA expression profiles show differential regulatory effects of microRNAs between estrogen receptor-positive and estrogen receptor-negative breast cancer. Genome Biol, 2009. 10(9): p. R90.

72. Loginov, V.I., et al., Novel miRNA genes hypermethylated in breast cancer. Molecular Biology, 2016. 50(5): p. 705-709.

73. Jansson, M.D. and A.H. Lund, MicroRNA and cancer. Molecular Oncology, 2012. 6(6): p. 590-610.

74. Mattiske, S., et al., The oncogenic role of miR-155 in breast cancer. Cancer Epidemiol Biomarkers Prev, 2012. 21(8): p. 1236-43.

75. Sochor, M., et al., Oncogenic microRNAs: miR-155, miR-19a, miR-181b, and miR-24 enable monitoring of early breast cancer in serum. BMC Cancer, 2014. 14: p. 448.

76. Chen, J., et al., Predicting distant metastasis and chemoresistance using plasma miRNAs. Medical Oncology, 2014. 31(1). 
77. Wang, L.Q., et al., miR-372 and miR-373 enhance the stemness of colorectal cancer cells by repressing differentiation signaling pathways. Molecular Oncology, 2018. 12(11): p. 1949-1964.

78. Wang, L., et al., MicroRNA-373 Inhibits Cell Proliferation and Invasion via Targeting BRF2 in Human Non-small Cell Lung Cancer A549 Cell Line. Cancer Res Treat, 2017.

79. Wei, F., et al., Diverse functions of miR-373 in cancer. J Transl Med, 2015. 13: p. 162.

80. Swellam, M., et al., Potential diagnostic role of circulating MiRNAs in breast cancer: Implications on clinicopathological characters. Clinical Biochemistry, 2018. 56: p. 47-54.

81. Müller, V., et al., Changes in serum levels of miR-21, miR-210, and miR-373 in HER2-positive breast cancer patients undergoing neoadjuvant therapy: a translational research project within the Geparquinto trial. Breast Cancer Res Treat, 2014. 147(1): p. 61-8.

82. Keklikoglou, I., et al., MicroRNA-520/373 family functions as a tumor suppressor in estrogen receptor negative breast cancer by targeting NF-KB and TGF- $\beta$ signaling pathways. Oncogene, 2012. 31(37): p. 4150-4163.

83. Guo, Y.H., et al., Down-regulation of miR-373 increases the radiosensitivity of lung cancer cells by targeting TIMP2. International Journal of Biochemistry \& Cell Biology, 2018. 99: p. 203-210.

\section{Tables}

Table 1. The Clinicopathological Characteristics 


\begin{tabular}{|c|c|c|c|}
\hline \multirow[t]{3}{*}{ Characteristic } & \multicolumn{2}{|l|}{ No of Patients (\%) } & \multirow[t]{3}{*}{$\mathrm{p}$} \\
\hline & \multicolumn{2}{|l|}{$N=34$} & \\
\hline & Before treatment & After NACT & \\
\hline \multicolumn{4}{|c|}{ Stage of cancer } \\
\hline I & $0(0.0)$ & $1(2.9)$ & \multirow[t]{6}{*}{0.563} \\
\hline$\| \mathrm{A}$ & $4(11.8)$ & $8(23.5)$ & \\
\hline IIB & $13(38.2)$ & $8(23.5)$ & \\
\hline IIIA & $9(26.5)$ & $8(23.5)$ & \\
\hline IIIB & $7(20.6)$ & 7 (20.7) & \\
\hline IIIC & $1(2.9)$ & $2(5.9)$ & \\
\hline $\mathrm{N}$-negative & $4(11.8)$ & $10(29.4)$ & \multirow[t]{2}{*}{0.289} \\
\hline $\mathrm{N}$-positive & $30(88.2)$ & $24(70.6)$ & \\
\hline \multirow[t]{2}{*}{ Index Ki 67} & 24.8 & 25.9 & \multirow[t]{2}{*}{0.855} \\
\hline & (16.60 to 42.60 ) & (15.03 to 39.10$)$ & \\
\hline
\end{tabular}

Table 2. The Levels of MRNA From Primary Tumor, After NACT and Adjacent Non-tumor Tissues 


\begin{tabular}{|c|c|c|c|c|c|}
\hline \multirow[t]{4}{*}{ miRNAs } & \multirow[t]{4}{*}{$\mathrm{n}$} & \multirow{2}{*}{\multicolumn{3}{|c|}{$\begin{array}{l}\text { Expression level of mRNA } \\
\text { Median }(25-75 \mathrm{P})\end{array}$}} & \multirow[t]{4}{*}{$\mathrm{p}$} \\
\hline & & & & & \\
\hline & & \multicolumn{2}{|l|}{ tumor tissue } & \multirow{2}{*}{$\begin{array}{l}\text { adjacent non-tumor } \\
\text { tissue } \\
n=15\end{array}$} & \\
\hline & & Before treatment & After NACT & & \\
\hline \multirow[t]{2}{*}{ miR-34a } & \multirow[t]{2}{*}{34} & 0.814 & 0.688 & 0.933 & \multirow[t]{2}{*}{0.733} \\
\hline & & $0.296-2.144$ & $0.144-4.000$ & $0.435-2.841$ & \\
\hline \multirow{2}{*}{$\begin{array}{l}\text { miR- } \\
124 a\end{array}$} & \multirow[t]{2}{*}{32} & $0.00325^{\star}$ & $0.0192^{\star \star}$ & 0.149 & $0.001 *$ \\
\hline & & $0.000623-0.0170$ & $0.00337-0.107$ & $0.0266-0.616$ & $0.004^{\star *}$ \\
\hline \multirow[t]{2}{*}{ miR-137 } & \multirow[t]{2}{*}{29} & 0.0012 & 0.000213 & 0.00052 & \multirow[t]{2}{*}{0.146} \\
\hline & & $0.000206-0.0204$ & $\begin{array}{l}0.0000396- \\
0.00176\end{array}$ & $0.0000256-0.00419$ & \\
\hline \multirow[t]{2}{*}{ miR-155 } & \multirow[t]{2}{*}{34} & 0.789 & 0.407 & 0.707 & \multirow[t]{2}{*}{0.741} \\
\hline & & $0.177-5.657$ & $0.0884-7.464$ & $0.278-2.327$ & \\
\hline \multirow[t]{2}{*}{ miR-373 } & \multirow[t]{2}{*}{29} & 0.000488 & $0.0000678^{*}$ & 0.00378 & \multirow[t]{2}{*}{$0.006 *$} \\
\hline & & $\begin{array}{l}0.0000405- \\
0.00427\end{array}$ & $\begin{array}{l}0.00000915- \\
0.00112\end{array}$ & $0.00159-0.0110$ & \\
\hline
\end{tabular}

* - the difference from the group with non-tumor tissue, $p<0.05$

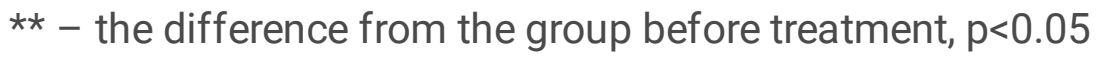

Table 3. Assosiciation Among Expression Levels of MicroRNAs and Histological Tumor Grades 
miRNAs Histological tumor grade

Median (25 - $75 \mathrm{P})$

\begin{tabular}{lllll} 
& Grade $1(\mathrm{n}=4)$ & Grade $2(\mathrm{n}=19)$ & Grade $3(\mathrm{n}=8)$ & \\
\hline $\begin{array}{l}\text { miR- } \\
\text { 34a }\end{array}$ & 0.988 & 0.574 & 0.912 & 0.726 \\
& $(0.798$ to 1.311$)$ & $(0.224$ to 1.961$)$ & $(0.282$ to 13.126$)$ & \\
\hline $\begin{array}{l}\text { miR- } \\
124 a\end{array}$ & 0.00995 & 0.00148 & 0.00225 & 0.712 \\
& $(0.00137$ to 0.110$)$ & $(0.00045$ to 0.0116$)$ & $(0.00148$ to 0.0101$)$ & \\
\hline miR- & $0.00115(0.00037$ to & $0.000977(0.0000835$ to & 0.000488 & 0.908 \\
137 & $0.00192)$ & $0.0139)$ & $(0.000166$ to 0.285$)$ & \\
\hline miR- & 0.308 & 0.467 & 1.66 & 0.392 \\
155 & $(0.177$ to 0.715$)$ & $(0.09$ to 3.258$)$ & $(0.265$ to 27.527$)$ & \\
\hline miR- & $0.00093(0.000247$ to & $0.000456(0.0000289$ to & $0.000431(0.000047$ to & 0.771 \\
373 & $0.0314)$ & $0.00527)$ & $0.000834)$ & \\
\hline
\end{tabular}

Table 4. Cox Proportional Analysis of the Prognostic Factors

\begin{tabular}{|llll|}
\hline Covariate & Coefficient of Cox model, b & Standard error, SE & $p$ \\
\hline miR-34a & -0.088 & 0.080 & 0.271 \\
\hline miR-124a & -48.0 & 46.6 & 0.303 \\
\hline miR-137 & -6.8 & 11.0 & 0.537 \\
\hline miR-155 & -0.19 & 0.26 & 0.465 \\
\hline miR-373 & -0.43 & 2.89 & 0.883 \\
Age & -0.039 & 0.027 & 0.147 \\
\hline Stage & 0.15 & 0.72 & 0.835 \\
\hline T & -0.04 & 0.36 & 0.914 \\
\hline N & -0.08 & 0.55 & 0.891 \\
\hline Ki-67 & 0.016 & 0.017 & 0.337 \\
\hline
\end{tabular}


Figures

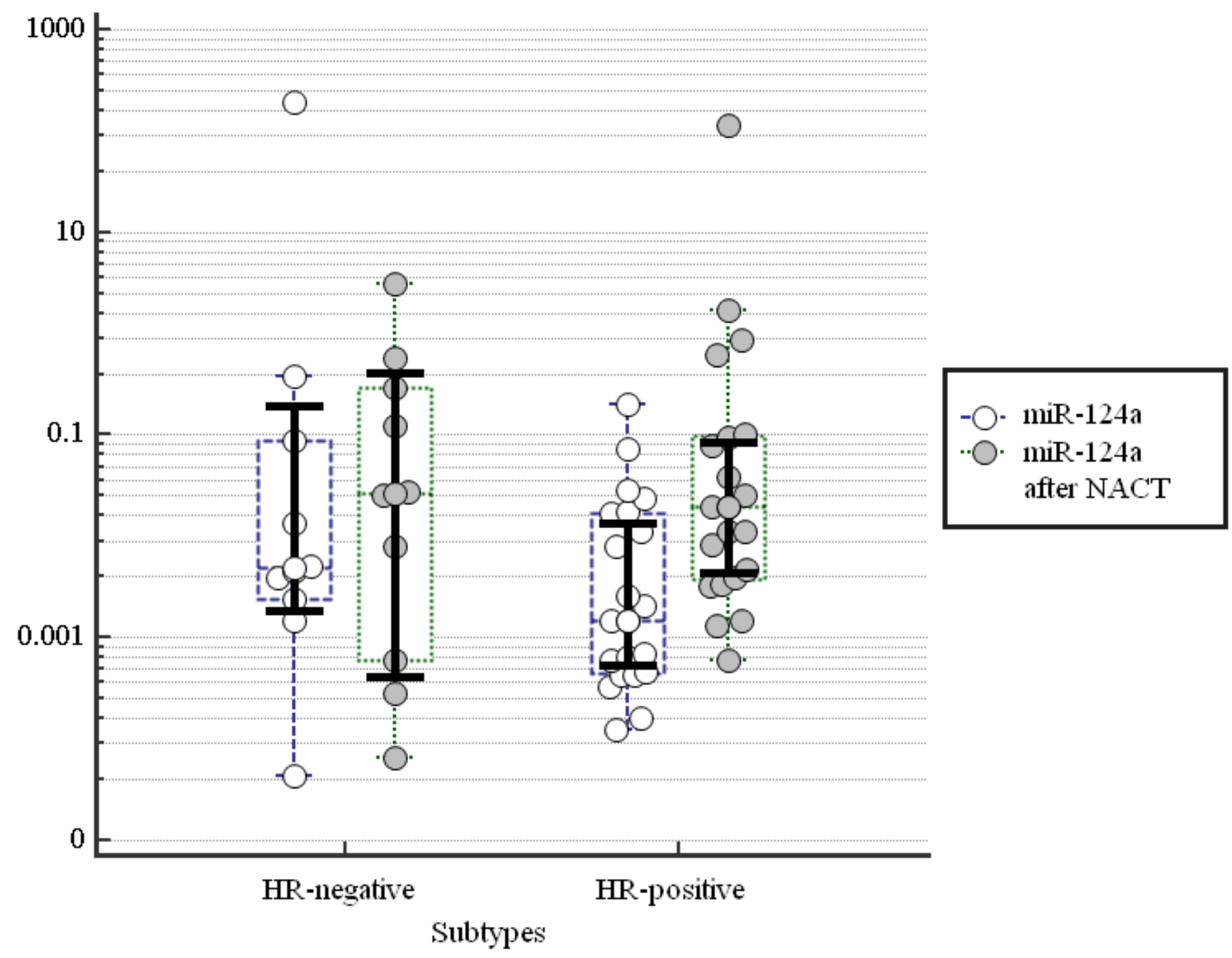

Figure 1

The expression of miR-124a in primary tumor tissue was increased after treatment with patients HRpositive $B C(p<0.05)$ 


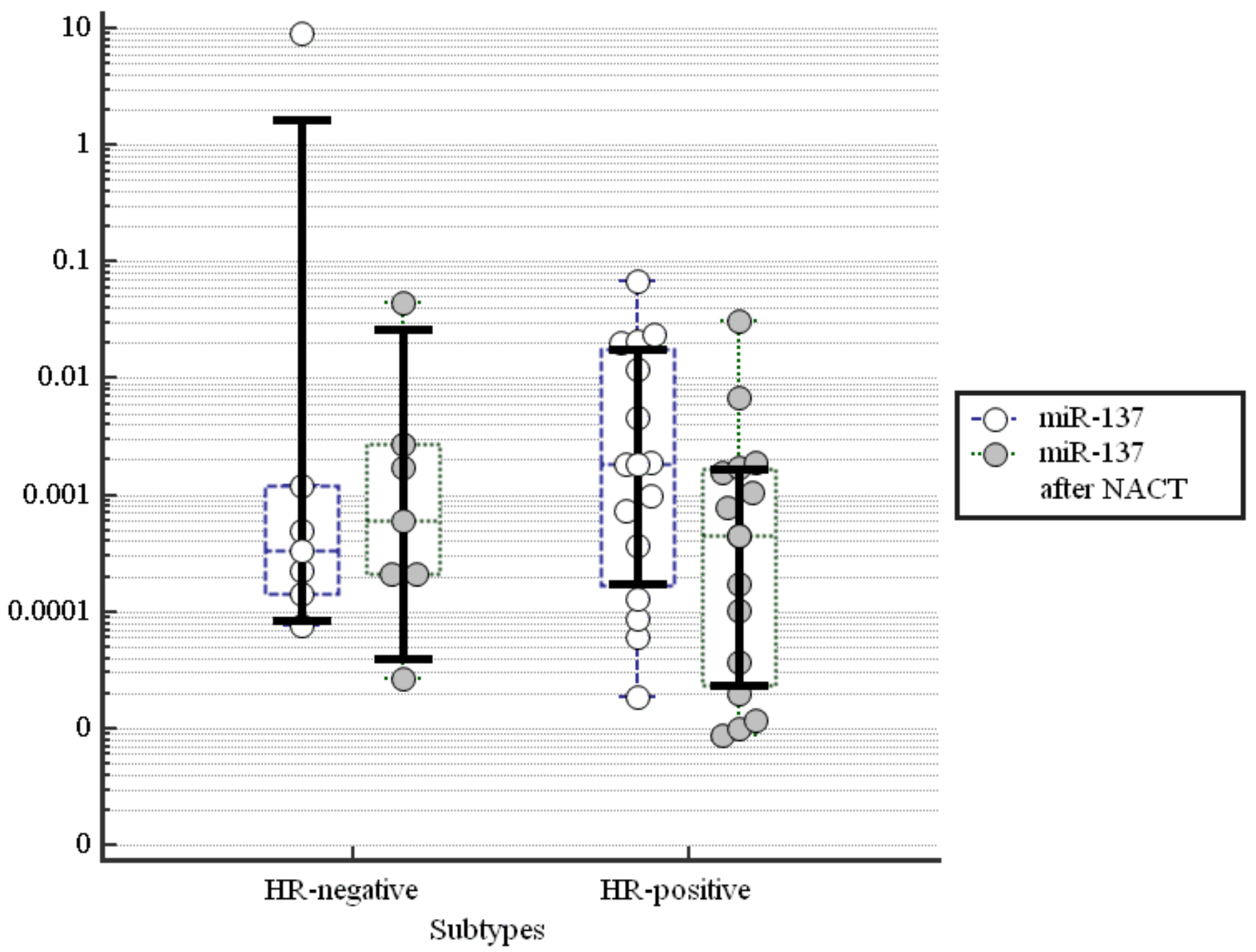

Figure 2

The expression of miR-137 in primary cancer tissues was decreased after treatment with patients HRpositive $B C(p<0.05)$ 